\title{
FOREWORD
}

\section{Special Section on Internet Technology VI}

The digital information and communication infrastructure using the Internet technology has been already a dependable information infrastructure platform of human activities and of business environment. After the deployment of as ADSL network, we are introducing a wireless LAN and FTTH (Fiber To The Home). The digital transmission technology to interconnect the digital devices to the Internet has been drastically changed in these days. This can be (1) "peer-to-peer" architecture from "client-server" architecture, (2) "No-more dial-up", i.e., so called the "native Internet" and (3) mobile nodes are larger number than of fixed nodes. As the result, the Internet becomes the digital communication platform internetworking all of digital devices using wide variety of digital transmission technologies. The Internet has been still grown more than exponentially in terms of the number of nodes, routing entries and traffic volume.

The research, development and deployment of internet technologies is based on a kind of positive closed and spiral feed back system. Internet protocol suit has been always modified, based on the operational experiences at the live Internet. This means that the internet technologies are highly engineering oriented. Actually, a lot of Internet researchers and engineers do not believe the new technology without "running code". A lot of research and development related with the Internet is based on the real operational experiences, and the new technologies are evaluated and appreciated by the Internet community itself.

Based on the above observation, this special issue is focusing on the practical works related with the internet technologies. Our paper accepting policy is; we accept the paper, when the work is very worth for the Internet community, even when it does not have the detailed analysis and evaluation. This particular special issue accommodates 14 papers, that should satisfy the above criteria. We have received about 50 paper submissions, from various countries, such as China, Taiwan or Korea.

Finally, it is really appreciated the hard work by guest editors and reviewers. Especially, all of guest editors want to express huge appreciation to Ms. Fumi Takahashi and Dr. Kenichi Nagami, who have managed all of administrative work.

Editorial Committee:

Secretary: Kenichi Nagami (INTEC NetCore Inc.)

Guest Editors: Atsushi Shionozaki (Sony CSL, Inc.), Ernst Thierry (Keio Univ.), Hideaki Imaizumi (Keio Univ.), Kaori Maeda (Hiroshima City Univ.), Katsuyuki Yamazaki (KDDI Lab.), Kengo Nagahashi (Univ. Tokyo), Kenji Watanabe (Saga Univ.), Koji Okamura (Kyushu Univ.), Masato Tsuru (Kyushu Institute of Technology), Motonori Nakamura (Kyoto Univ.), Ray Atarashi (IIJ), Ryuji Wakikawa (Keio Univ.), Suguru Yamaguchi (NAIST), Tomohiro Fujisaki (NTT), Youki Kadobayashi (NAIST), Yutaka Nakamura (NAIST)

Hiroshi Esaki, Guest Editor-in-Chief

Hiroshi Esaki (Member) Ph.D., Professor, The University of Tokyo, Executive Director for Japan IPv6 promotion council, Board member for WIDE project, Director of JPNIC (Japan Network Information Center). He received the B.E. and M.E. degrees from Kyushu University, Fukuoka, Japan, in 1985 and 1987, respectively. And, he received Ph.D. from University of Tokyo, Japan, in 1998. In 1987, he joined Research and Development Center, Toshiba Corporeation, where he engaged in the research of ATM systems. From 1990 to 1991, he has been at Applied Research Laboratory of Bellcore Inc., New Jersey (USA), as a residential researcher. From 1994 to 1996, he has been at CTR (Center for Telecommunication Research) of Columbia University in New York (USA). During his staying at Columbia University, he has proposed the CSR architecture, that is the origin of MPLS(Multi-Protocol Label Switching), to the IETF and to the ATM Forum. From 1996 to 1998, he has conducted the CSR project in Toshiba, as a chief architect. From 1998, he has served as a professor at the University of Tokyo, and as a board member of WIDE Project (www.wide.ad.jp). 